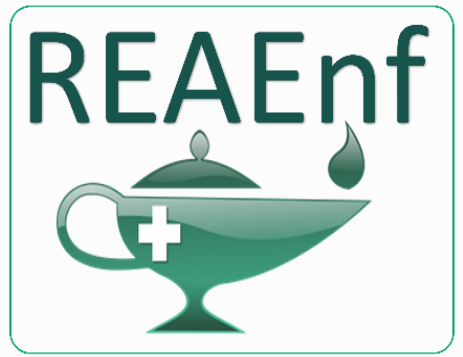

Revista Eletrônica Acervo Enfermagem

\section{REVISÃO BIBLIOGRÁFICA}

Recebido em: 9/2020

Aceito em: 10/2020

Publicado em: 11/2020

\title{
A importância do tempo para o clampeamento do cordão umbilical para o recém-nascido: uma revisão integrativa da literatura
}

\author{
The importance of time for clamping the umbilical cord for the newborn: an integrative \\ literature review
}
La importancia del tiempo para sujetar el cordón umbilical para el recién nacido: una revisión integrativa de la literatura

\begin{abstract}
Bárbara Alves Ruela de Azevedo Ruivo ${ }^{1}$, Jéssica Paloma Cardoso Bastos ${ }^{1}$, Adilson Mendes de Figueiredo Júnior ${ }^{2 *}$, Jamilly Cristina da Silva Silva², Élen Gabriela Sales Costa ${ }^{2}$, Dulce Quadros Pereira $^{2}$, Letícia Figueira dos Santos ${ }^{2}$, Christian Boaventura dos Santos ${ }^{3}$, Giovanna Farias de Sousa $^{4}$, Edeiza Priscila de Souza Pinheiro ${ }^{5}$.
\end{abstract}

\begin{abstract}
Resumo: Esse artigo buscou analisar o impacto e a importância do tempo do clampeamento do cordão umbilical para o recém-nascido. Tratou-se de uma revisão integrativa da literatura realizada na plataforma eletrônica Biblioteca Virtual em Saúde (BVS) que compõem as bases de dados: PubMed, Lilacs, Scielo e Medline. Após a análise da amostra observou-se abordagens distintas em relação ao momento adequado para clampeamento do cordão umbilical, no entanto $60 \%$ dos estudos evidenciaram diversos benefícios do clampeamento tardio quando comparado ao precoce. Sendo identificadas três temáticas principais: Fatores positivos relacionados ao clampeamento tardio; Fatores relacionados ao clampeamento precoce e a importância da enfermagem na prática do clampeamento tardio. Na literatura ainda se tem opiniões e resultados científicos divergentes sobre o tempo ideal para a realização do clampeamento do cordão umbilical, no entanto às evidenciais dos benefícios a curto e em longo prazo para o bebê e para a mãe. Atualmente se recomenda a realização do clampeamento tardio e os resultados encontrados nesse estudo reforçam a importância de tal prática. Porém, este estudo identificou a escassez de publicações acerca do clampeamento tardio disponíveis.
\end{abstract}

Palavras-chave: Enfermagem, Cordão umbilical, Constrição.

Abstract: This article sought to analyze the impact and the importance of the time of clamping of the umbilical cord for the newborn. It was an integrative literature review conducted on the electronic platform Virtual Health Library (VHL) that make up the databases: PubMed, Lilacs, Scielo and Medline. After

\footnotetext{
${ }^{1}$ Centro Universitário Maurício de Nassau (UNINASSAU), Belém - Pará.

2 Escola Superior da Amazônia (ESAMAZ), Belém - Pará. *E-mail: adilsonmdfj@hotmail.com

3 Universidade Federal do Pará (UFPA), Belém - Pará.

${ }^{4}$ Universidade do Estado do Pará (UEPA), Belém - Pará.

${ }^{5}$ Centro Universitário do Pará (CESUPA), Belém - Pará.
} 
analyzing the sample, different approaches were observed in relation to the appropriate time for clamping the umbilical cord, however $60 \%$ of the studies showed several benefits of late clamping when compared to the early clamping. Three main themes were identified: Positive factors related to late clamping; Factors related to early clamping and the importance of nursing in the practice of late clamping. In the literature, there are still divergent opinions and scientific results about the ideal time to perform the clamping of the umbilical cord, however the evidences of the short and long term benefits for the baby and the mother. Currently, late clamping is recommended and the results found in this study reinforce the importance of such practice. However, this study identified the scarcity of publications on late clamping available.

Keywords: Nursing, Umbilical cord, Constriction.

Resumen: Este artículo buscó analizar el impacto y la importancia del tiempo de pinzamiento del cordón umbilical para el recién nacido. Se trató de una revisión integradora de la literatura realizada en la plataforma electrónica Virtual Health Library (BVS) que componen las bases de datos: PubMed, Lilacs, Scielo y Medline. Luego de analizar la muestra, se observaron diferentes abordajes en relación al tiempo apropiado para pinzar el cordón umbilical, sin embargo el $60 \%$ de los estudios mostró varios beneficios del pinzamiento tardío en comparación con el pinzamiento temprano. Se identificaron tres temas principales: factores positivos relacionados con el pinzamiento tardío; Factores relacionados con el pinzamiento temprano y la importancia de la enfermería en la práctica del pinzamiento tardío. En la literatura, aún existen opiniones divergentes y resultados científicos sobre el momento ideal para realizar el pinzamiento del cordón umbilical, sin embargo las evidencias de los beneficios a corto y largo plazo para el bebé y la madre. Actualmente se recomienda el pinzamiento tardío y los resultados encontrados en este estudio refuerzan la importancia de dicha práctica. Sin embargo, este estudio identificó la escasez de publicaciones disponibles sobre pinzamiento tardío.

Palabras clave: Enfermería, Cordón umbilical, Constricción.

\section{INTRODUÇÃO}

Sabe-se que o momento do parto e do pós-parto imediato são períodos de grande vulnerabilidade tanto para mãe quanto o para o recém- nascido. Por essa razão se faz necessário que todos os procedimentos e intervenções realizados, sejam pensados e analisados criticamente, visando promover benefícios a curtos e longo prazo para a saúde de ambos. Atualmente, se observa que o clampeamento do cordão umbilical, muitas vezes é realizado de maneira mecânica e imediata ao parto sem que se tenha um pensamento reflexível e critico de tal conduta sobre os possíveis impactos futuros para o bebê (WHO, et al., 2013).

O clampeamento imediato do cordão umbilical começou a ser utilizado quando o parto hospitar começou a ser visto como um parto seguro e se baseou no atendimento rápido ao recém-nascido e na prevenção da hemorragia materna no pós-parto, no entanto as praticas atuais baseadas no entendimento do processo fisiológico defendem o clampeamento tardio apesar de tal discursão persistir até os dias atuais (MONDINI L, et al., 2010).

A prática do clampeamento tardio do cordão umbilical é proveniente de recomendações das áreas de obstetrícia e neonatologia e se discute desde 1801, por Erasmus Darwin um dos percursores a se opor ao clampeamento imediato, considerando que deveria esperar que se cessassem as pulsações do cordão, caso contrário, o recém-nascido se prejudicaria tendo em vista, que parte do sangue que sai da placenta deveria pertencer a ele. Posteriormente, Pierre Budin considerado o precursor da neonatologia, em 1875 publicou um artigo intitulado "Quando devemos clampear o cordão umbilical" alegando que o volume de sangue retido na placenta após o clampeamento precoce do cordão umbilical era impedido de ser transferido ao neonato devido o clampeamento precoce (WHO, et al., 2013). Considera-se como clampeamento tardio aquele realizado após um a três minutos do nascimento ou assim que cessar as pulsações do cordão umbilical.

REAEnf/EJNC | Vol. 4 | e5220 | DOI: https://doi.org/10.25248/REAenf.e5220.2020 Página 2 de 9 
Observa-se que na literatura ainda não se tem um consenso e evidencias suficientes para estabelecimento de um tempo especifico para a realização do clampeamento e de seus impactos negativos na saúde do recém-nascido (MONDINI L, et al., 2010).

No entanto, artigos e estudos evidenciam que o clampeamento precoce acarreta na diminuição dos estoques de ferro da criança, diminuição da concentração de hemoglobina aos dois e três meses de idade e pode provocar o aumentando do risco de anemia em crianças a termo, enquanto que o tardio aumenta o volume de hemácias no bebê, podendo, consequentemente, elevar os níveis de bilirrubina consequentemente reduzindo a anemia infantil e a hemorragia intraventricular, reduzindo a enterocolite necrosante e sepse infantil (WHO, et al., 2013).

Desse modo, o presente artigo busca analisar o que a literatura dos últimos 5 anos nos mostra a cerca da importância do tempo para o clampeamento do cordão umbilical e os benefícios dessa prática aos neonatos.

\section{MÉTODOS}

O estudo trata-se de uma revisão integrativa da literatura que consiste em um método de pesquisa que permiti buscar, analisar criticamente, e sintetizar evidências presentes na literatura acerca do tema pesquisado, resultando em um estudo representativo do estado do conhecimento atual da temática abordada, além disso, possibilita encontrarmos lacunas existentes no cenário cientifico que nos direcionam para o desenvolvimento de futuras pesquisar e intervenções.

A realização desta revisão integrativa ocorreu em 6 (seis) etapas que consistiu na escolha do tema e as questões de pesquisa, estabelecimento de critérios para inclusão e exclusão de estudo, avaliação dos estudos incluídos, definição das informações a serem extraídas e utilizadas dos estudos selecionados, interpretação dos resultados e a síntese do conhecimento.

Nesta revisão, a coleta de dados foi realizada no período de Setembro a Outubro de 2019, utilizando artigos e trabalhos científicos disponíveis na plataforma eletrônica Biblioteca Virtual em Saúde (BVS) que compõem as bases de dados: PubMed, Literatura Latino-Americana e do Caribe em Ciências da Saúde (Lilacs), Scientific Electronic Library Online (Scielo) e Medical Literature Analysis and Retrievel System Online (Medline).

Utilizou-se os seguintes descritores: constrição and cordão umbilical e constrição and cordão umbilical and enfermagem, tais descritores foram considerados como mais apropriados para a pesquisa após leituras previas de artigos abordando a temática pesquisada e a utilização da plataforma DeCS.

Foram utilizados como critérios de inclusão para esta pesquisa os trabalhos científicos disponíveis na integra na internet, no idioma português do Brasil, inglês e espanhol, publicados no período de 2015 a 2019 e como critérios de exclusão os trabalhos indisponíveis na integra, duplicados nas bases de dados, os que não correspondiam ou se enquadravam à temática abordada, os publicados fora do período determinado para a pesquisa e os que não respondiam a questão norteadora do estudo: "Qual a importância do tempo para o clampeamento do cordão umbilical do recém-nascido?".

Após a análise dos critérios de inclusão e exclusão, a leitura do titulo e do resumo, foram selecionados 36 artigos para serem lidos na integra, no entanto, alguns deles não foram condizentes com a necessidade do estudo, resultando em apenas 9 (nove) artigos selecionados, conforme observamos no Quadro 1.

Por fim, foi utilizado um instrumento de coleta de dados contendo a identificação do artigo (título, objetivo, método, pais e ano de publicação e principais resultados e recomendações/conclusões) com o intuito de facilitar e organizar o entendimento sobre o conteúdo dos artigos selecionados.

REAEnf/EJNC | Vol. 4 | e5220 | DOI: https://doi.org/10.25248/REAenf.e5220.2020 Página 3 de 9 
Quadro 1 - Distribuição dos artigos científicos segundo base de dados e descritores, 2019.

\begin{tabular}{|c|c|c|c|}
\hline Descritores & Base de dados & $\begin{array}{c}\text { Artigos } \\
\text { encontrados }\end{array}$ & $\begin{array}{c}\text { Artigos } \\
\text { selecionados }\end{array}$ \\
\hline \multirow{3}{*}{ Constrição AND cordão umbilical } & $\begin{array}{l}\text { PUBMED e } \\
\text { MEDLINE }\end{array}$ & 112 & 8 \\
\hline & LILACS & - & - \\
\hline & SCIELO & - & - \\
\hline \multirow{3}{*}{$\begin{array}{c}\text { Constrição AND cordão umbilical } \\
\text { AND Enfermagem }\end{array}$} & $\begin{array}{l}\text { PUBMED e } \\
\text { MEDLINE }\end{array}$ & 5 & 1 \\
\hline & LILACS & - & - \\
\hline & SCIELO & - & - \\
\hline \multicolumn{2}{|l|}{ Total } & 117 & 09 \\
\hline
\end{tabular}

Fonte: Ruivo BARA, et al., 2019.

\section{RESULTADOS}

Os nove (9) artigos encontrados na pesquisa são de língua inglesa. Observou-se que no ano de 2019 não foi encontrada publicações nas bases de dados e bibliotecas científicas utilizadas no estudo.

A seguir serão apresentados os demais dados da amostra por meio do Quadro 2, no qual estão sintetizadas informações a fim de atender o objetivo e a questão norteadora deste estudo. Os elementos apresentados são o título do artigo, o nome dos autores, o ano de publicação, os objetivos e tipo de estudo bem como as conclusões de cada artigo. 
Quadro 2 - Síntese dos resultados da pesquisa, 2019.

\begin{tabular}{|c|c|c|c|c|}
\hline Título do Artigo & Autores & Ano & Objetivo & Tipo de estudo \\
\hline $\begin{array}{l}\text { Clampeamento tardio versus imediato do } \\
\text { cordão umbilical em bebês prematuros. }\end{array}$ & $\begin{array}{l}\text { Tarnow-Mordi W, et } \\
\text { al. (2017) }\end{array}$ & 2017 & $\begin{array}{l}\text { Analisar o momento preferido da pinça do cordão umbilical } \\
\text { em prematuros }\end{array}$ & $\begin{array}{l}\text { Estudo piloto randomizado } \\
\text { de ensaio clínico. }\end{array}$ \\
\hline $\begin{array}{l}\text { Clampeamento tardio vs precoce do } \\
\text { cordão umbilical para bebês prematuros: } \\
\text { uma revisão sistemática e meta-análise. }\end{array}$ & $\begin{array}{l}\text { Tarnow-Mordi W, et } \\
\text { al. (2017) }\end{array}$ & 2017 & $\begin{array}{c}\text { Comparar os efeitos do clampeamento tardio vs do cordão } \\
\text { precoce na mortalidade hospitalar (desfecho primário) e } \\
\text { morbidade em prematuros. }\end{array}$ & Ensaio clínico randomizado. \\
\hline $\begin{array}{l}\text { Resultados clínicos em bebês } \\
\text { prematuros após a instituição de uma } \\
\text { mudança na prática de pinçamento do } \\
\text { cordão umbilical com retardo. }\end{array}$ & $\begin{array}{l}\text { Fenton C, et al. } \\
\qquad(2018)\end{array}$ & 2018 & $\begin{array}{c}\text { Avaliar a viabilidade clínica, a eficácia e os resultados de } \\
\text { segurança em prematuros que receberam DCC após uma } \\
\text { implementação de mudança de prática destinada a reduzir a } \\
\text { incidência de HIV. }\end{array}$ & $\begin{array}{l}\text { Estudo prospectivo, } \\
\text { mascarado, randomizado, } \\
\text { controlado. }\end{array}$ \\
\hline $\begin{array}{l}\text { Associação entre estresse oxidativo e } \\
\text { lipídios séricos do cordão em relação ao } \\
\text { retardo do clampeamento do cordão em } \\
\text { neonatos a termo. }\end{array}$ & $\begin{array}{c}\text { Moustafa AN, et al. } \\
(2017)\end{array}$ & 2017 & $\begin{array}{l}\text { Avaliar a influência do clampeamento precoce ou tardio do } \\
\text { estresse oxidativo em recém-nascidos a termo e avaliar a } \\
\text { associação de estresse oxidativo e lipídios no sangue do } \\
\text { cordão umbilical }\end{array}$ & $\begin{array}{c}\text { Estudo transversal } \\
\text { observacional analítico. }\end{array}$ \\
\hline $\begin{array}{l}\text { Efeito da fixação tardia do cordão } \\
\text { umbilical no fluxo sanguíneo sistêmico: } \\
\text { um ensaio clínico randomizado. }\end{array}$ & $\begin{array}{l}\text { POPAT H, et al. } \\
(2016)\end{array}$ & 2016 & $\begin{array}{c}\text { Determinar se o pinçamento tardio do cordão melhora o fluxo } \\
\text { sanguíneo sistêmico em comparação com o pinçamento } \\
\text { imediato do cordão umbilical em bebês muito prematuros nas } \\
\text { primeiras } 24 \text { horas. }\end{array}$ & Ensaio clínico randomizado. \\
\hline $\begin{array}{l}\text { Parteiras na Índia: uma intervenção de } \\
\text { pinçamento retardado do cordão } \\
\text { umbilical usando simulação. }\end{array}$ & $\begin{array}{l}\text { Faucher MA, et al. } \\
\qquad(2016)\end{array}$ & 2016 & $\begin{array}{c}\text { O objetivo do estudo foi aumentar a utilização do } \\
\text { clampeamento tardio do cordão por um grupo de parteiras } \\
\text { que trabalha em Hyderabad, na Îndia. }\end{array}$ & $\begin{array}{l}\text { Estudo qualitativo } \\
\text { explorativo. }\end{array}$ \\
\hline $\begin{array}{c}\text { Clampeamento retardado do cordão } \\
\text { umbilical no século } 21 \text { : indicações para a } \\
\text { prática }\end{array}$ & Bayer K (2016) & 2016 & $\begin{array}{l}\text { O objetivo deste artigo é discutir a fisiologia do pinçamento do } \\
\text { cordão umbilical, os benefícios potenciais e os efeitos } \\
\text { adversos do pinçamento tardio do cordão umbilical, e como } \\
\text { isso afeta a enfermeira da prática avançada. }\end{array}$ & Pesquisa Bibliográfica. \\
\hline $\begin{array}{c}\text { Efeito do clampeamento retardado do } \\
\text { cordão no neuro desenvolvimento aos } 4 \\
\text { anos de idade: um ensaio clínico } \\
\text { randomizado. }\end{array}$ & $\begin{array}{l}\text { Andersson O, et al. } \\
(2015)\end{array}$ & 2015 & $\begin{array}{l}\text { Investigar os efeitos do clampeamento tardio em comparação } \\
\text { com a clampeamento precoce no neurodesenvolvimento aos } \\
\qquad 4 \text { anos de idade. }\end{array}$ & Ensaio clínico randomizado. \\
\hline $\begin{array}{l}\text { Clampeamento tardio do cordão } \\
\text { umbilical em neonatos sul-africanos com } \\
\text { baixo peso ao nascer esperado: um } \\
\text { ensaio clínico randomizado. }\end{array}$ & $\begin{array}{l}\text { Tiemersma S, et al. } \\
(2015)\end{array}$ & 2015 & $\begin{array}{c}\text { Avaliar os efeitos hematológicos e de segurança do } \\
\text { pinçamento tardio do cordão umbilical em bebês com baixo } \\
\text { peso ao nascer esperado em um ambiente com poucos } \\
\text { recursos. }\end{array}$ & $\begin{array}{l}\text { Estudo randomizado } \\
\text { controlado. }\end{array}$ \\
\hline
\end{tabular}

Fonte: Ruivo BARA, et al., 2019.

REAEnf/EJNC | Vol. 4 | e5220 | DOI: https://doi.org/10.25248/REAenf.e5220.2020 Página $\mathbf{5}$ de 9 
No Quadro 3 foram apresentados os principais benefícios do clampeamento tardio do cordão umbilical nos recém-nascidos identificados nos artigos científicos utilizados como amostra deste estudo.

Quadro 3 - Benefícios do clampeamento tardio do cordão umbilical de acordo com a literatura, 2019.

\begin{tabular}{|l|c|c|}
\hline \multicolumn{1}{|c|}{ Efeitos do clampeamento tardio } & Ano & Autores \\
\hline $\begin{array}{l}\text { - Redução da mortalidade hospitalar; } \\
\text { - Redução da incidência de baixo índice de Apgar em 1 minuto; } \\
\text { - Elevação do pico de hematócrito; } \\
\text { - Redução da proporção de crianças com transfusão de sangue } \\
\text { em 10\%. }\end{array}$ & 2017 & $\begin{array}{c}\text { Tarnow-Mordi W, et al. } \\
\text { (2017) }\end{array}$ \\
\hline $\begin{array}{l}\text { - Redução do risco de HIV; } \\
\text { - Para crianças com 29 ou menos semanas de idade gestacional, } \\
\text { a temperatura axilar na admissão foi significativamente maior } \\
\text { naquelas que receberam clampemento tardio; } \\
\text { - Bebês com mais de 29 semanas de idade gestacional que } \\
\text { receberam DCC apresentaram escores Apgar de 1 minuto, } \\
\text { temperatura e nível de bilirrubina de 24 horas significativamente } \\
\text { mais altos. }\end{array}$ & 2018 & Fenton C, et al. (2018) \\
\hline $\begin{array}{l}\text { - Diminuição dos lipídios no sangue e a uma atividade } \\
\text { antioxidante aumentada. }\end{array}$ & 2017 & Moustafa NA, et al. (2017) \\
\hline $\begin{array}{l}\text { - Diminuição da frequência de anemia por deficiência de ferro no } \\
\text { primeiro ano de vida; } \\
\text { - Melhores resultados no desenvolvimento neurológico em recém- } \\
\text { nascidos a termo; } \\
\text { - Redução de transfusões de sangue, possível transfusão } \\
\text { autóloga de células-tronco e na incidência de hemorragia } \\
\text { intraventricular. }\end{array}$ & 2016 & Bayer K (2016) \\
\hline $\begin{array}{l}\text { - Melhorou os escores nos domínios motor e social aos 4 anos de } \\
\text { idade. }\end{array}$ & 2015 & Andersson O, et al. (2015) \\
\hline
\end{tabular}

Fonte: Ruivo BARA, et al., 2019.

\section{DISCUSSÃO}

A partir da analise e interpretação dos resultados dos estudos conforme observado na tabela 2 observou-se que três (30\%) dos estudos analisados que não houveram diferenciações ou benefícios do clampeamento tardio do cordão umbilical em comparação ao clampeamento precoce; seis (60\%) dos estudos evidenciaram diversos benefícios do clampeamento tardio quando comparado ao precoce, enquanto que apenas um (10\%) dos estudo concluiu que os possíveis danos do pinçamento tardio podem incluir a policitemia e hiperbilirrubinemia.

No estudo Bayer K (2016), foi evidenciado que o pinçamento atrasado reduziu a mortalidade hospitalar, reduziu a incidência de baixo índice de Apgar em 1 minuto, mas não em 5 minutos, constatou também que não reduziu a incidência de intubação para reanimação, porém aumentou o pico de hematócrito e reduziu a proporção de crianças com transfusão de sangue em 10\%, dados também evidenciados no estudo de Tiemersma S, et al. (2015).

Contudo, diferentemente do que foi encontrado, na pesquisa de Fenton C, et al. (2018); com recémnascidos com risco de HIV onde afirma que não houve diferença entre 1 e 5 minutos após o nascimento, nos nível de bilirrubina de 24 horas ou nível de hematócrito. Complementando os estudos acima Moustafa NA, et al. (2017), observou que clampeamento tardio foi associado a uma diminuição dos lipídios no sangue 
do cordão umbilical e a uma atividade antioxidante aumentada. Sugerindo o efeito protetor do clampeamento tardio na saúde futura do termo neonatos apoiando a aplicação do clampeamento tardio do cordão umbilical no gerenciamento ativo do terceiro estágio do trabalho de parto em neonatos a termos. Faucher MA, et al. (2016), mostram aumentos significativos no conhecimento e crenças positivas sobre a prática de pinçamento tardio do cordão umbilical. A simulação foi eficaz para obter feedback importante relacionado ao aprendizado.

Já os estudos que comparam o clampeamento tardio e o precoce, POPAT $\mathrm{H}$, et al. (2016) o clampeamento tardio do cordão não teve efeito no fluxo sanguíneo sistêmico, medido como o menor fluxo médio nas primeiras 24 horas em bebês com menos de 30 semanas de gestação. Tarnow-Mordi W, et al. (2017) complementa que entre os prematuros, o pinçamento tardio do cordão não resultou em menor incidência do resultado combinado de morte ou maior morbidade às 36 semanas de gestação do que o pinçamento imediato do cordão umbilical.

Andersson O, et al. (2015), apontam também que o clampeamento tardio em comparação com o clampeamento precoce melhorou os escores nos domínios motor e social aos 4 anos de idade, especialmente em meninos, indicando que a otimização do tempo para o CC pode afetar o neuro desenvolvimento em uma população de baixo risco de crianças nascidas em famílias de alta renda país.

Alguns ensaios clínicos randomizados e metanálises evidenciaram que o clampeamento tardio do cordão umbilical por pelo menos um minuto após o nascimento em recém-nascidos implica na passagem substancial de sangue referente da placenta para o bebê (transfusão placentária): aumentando em média o peso dos bebês acerca de $101 \mathrm{~g}$. Consequentemente, há um aumento na hemoglobina 48 horas após o nascimento e diminuindo assim a probabilidade de deficiência de ferro durante a infância. (MCDONALD SJ, et al., 2014).

Além de diminuir complicações futuras, o clampeamento tardio aumenta o canto materno-recém-nascido, proporcionando maior ligação entre a mãe e o bebê, por consequente, aumento do sucesso da amamentação. Isto de nada impede a realização fácil do clampeamento. Ressalta-se, porém, a suma importância da monitorização atenciosa e precisa dos sinais vitais dos bebês durante este processo (DAVANZO R, et al., 2015).

Vale ressaltar que mesmo que seja realizado o clampeamento tardio o manejo ativo do terceiro estágio do trabalho de parto não deve ser interrompido, abarcando o uso de agentes uterotônicos após o parto do recém-nascido para minimizar o sangramento materno (WHO, 2018).

A prática do clampeamento imediato é uma cultura hospitalar realizada de modo rotineiro principalmente devido a sugestões advindas dos profissionais das áreas de obstetrícia e neonatologia, sendo o principal argumento a prevenção de icterícia e policitemia (MONDINI L, et al., 2010).

Ou seja, uma prática realizada sem muitas evidências científicas. Apesar de diversas pesquisas produzidas acerca das vantagens do clampeamento tardio, ainda observa-se a prática da ligadura precoce, pelo simples aceleramento da separação do binômio mãe-RN na sala de parto ou também pela presença do médico pediatra na sala de parto, ansioso para prestar uma assistência ao recém-nascido o mais precoce possível (PHILIP AGS e SAIGAL S, 2004).

Em certos casos, a ligadura precoce do cordão ocorre quando há a necessidade de realizar a coleta de sangue do cordão umbilical e placentária (SCUP), havendo recomendações científicas baseadas em evidências, nos primeiros 30 segundos após a expulsão do feto, devido ao aumento de volume coletado (ARMSON BA, et al., 2005). Porém este decisão cabe unicamente e definitivamente a equipe multiprofissional envolvida no processo de parto, pois a prioridade é a manutenção da segurança materno e neonato (BERTOLINI F, et al., 1995). O Instituto Brasileiro de Segurança do Paciente (2019), coloca que o campleamento tardio prejudica significativamente no coleta do SCUP para os Centros de Processamento Celular (CPC), diminuindo o volume coletado juntamente com a contagem de células nucleadas das doações. Conclui-se que nos casos de famílias e pacientes doadores o bloqueio precoce contribui para o rendimento da bolsa.

REAEnf/EJNC | Vol. 4 | e5220 | DOI: https://doi.org/10.25248/REAenf.e5220.2020 Página 7 de 9 
Segundo o estudo de Chaparro CM, et al. (2006), desenvolvidos por uma década, no México, na África do Sul, na China e nos Estados Unidos os níveis de ferritina e os níveis de hemoglobina observando os seis primeiros meses de vida, não mostraram diferenças significativas nos níveis de hemoglobina entre crianças com clampeamentos precoce e tardio aos 3, 6 e 9 meses de idade. Outros pontos colocados pela Who, et al. (2013), para realização do campleamento precoce são: placenta não intacta, ou seja, anormal, desprendimento ou avulsão do cordão umbilical; como também a instabilidade hemodinâmica materna ou necessidade de RCPN.

O tipo de clampeamento realizado na sala de parto é um dos fatores determinantes das múltiplas causas para ocorrência de anemia em prematuros, pois diminui a presença de hematócritos. A ligadura tardia do cordão umbilical gera o aumento do fluxo sanguíneo após o nascimento, melhorando assim os números hematológicos (MCDONALD SJ, et al., 2014).

Contudo nos bloqueios precoces há uma diminuição da presença de hematrócitos necessitando de transfusão sanguínea, consequentemente internação em Unidade de Terapia Intensiva Neonatal, devido não ser um procedimento isento de risco, apresenta diversas complicações infecciosas e não infecciosas, necessitando de cuidados mais intensivos e específicos (CHOPARD MRT, et al., 2010).

Pesquisas apontam que quando realizado o clampeamento precoce do cordão umbilical, principalmente em casos que há necessidade de realizar a Reanimação Cardiopulmonar (RCP), o volume decaem de 30 até $50 \%$ menos de hemácias para o recém-nascido, podendo gerar uma pré-disposição para lesão isquêmica. A necessidade RCP neonatal (RCPN) gera, normalmente, o comprometimento do desenvolvimento cognitivo em crianças de 8 anos de idade. Dessa forma, faz-se de sua importância 0 registro em prontuário do pinçamento do cordão umbilical afim de estudos para verificar se há alterações fisiológicas na transição fetal-neonatal e assegurar que a prática da RCPN não cause nenhum tipo de dano (MERCER J e BEWLEY S, 2009). Visto que esse neonato terá que ser internado em Unidade de Terapia Intensiva Neonatal para garantir a recuperação mais rápida e sem pós-complicações.

Segundo Who, et al. (2013), há um pequeno aumento na presença de icterícia que necessita de fototerapia em recém-nascidos a termo que passam por bloqueio tardio. Por isso, são necessários que os profissionais que trabalham diretamente com essa estratégia, como obstetras e enfermeiros obstetras, assegurem um mecanismo para tratar a icterícia neonatal. Porém, estudos revelaram que não há subsídios suficientes para apontar o campleamento tardio como ponto crucial no aumento no número de internações em Unidade de Terapia Intensiva Neonatal, mas estima-se que com o passar dos anos, estudos mais específicos possam aprimorar essa dinâmica e atualizar os dados sobre o clampeamento (MATHEW JL, 2011).

\section{CONSIDERAÇÕES FINAIS}

A realização do clampeamento do cordão umbilical é um procedimento inevitável e na literatura ainda se encontram opiniões e resultados científicos divergentes sobre o tempo ideal para a realização de tal procedimento, porém as diretrizes nacionais e internacionais preconizam que o tempo seja de 30-60 segundos após o nascimento. A pesquisa mostrou que às evidenciais dos benefícios a curto e longo prazo para o binômio mãe-bebê quanto à realização do campleamento tardio, concomitantemente com que é preconizado pelas diretrizes. Estes resultados encontrados reforçam a importância da prática realizada, como também do conhecimento dos profissionais de saúde envolvidos em realizar tal manobra. Porém, este estudo identificou a escassez de publicações sobre o clampeamento precoce, disponíveis nos idiomas português e espanhol, nos anos de 2018 e 2019, não podendo assim fazer uma comparação colocando o tardio como benéfico e o precoce como prejudicial. A questão envolvendo os índices de internação em Unidades de Terapia Intensiva Neonatal quanto ao tempo à escolha do clampeamento, o estudo mostrou a correlação quanto ambas as estratégias podem acarretar na internação do recém nascido no setor de tratamento intensivo. Sendo assim, este estudo propõe como medida de intervenção o incentivo e a realização de pesquisas mais profundas e específicas com a finalidade de oferecer mais subsídios para constatação e comprovação do que é preconizado pelas diretrizes.

REAEnf/EJNC | Vol. 4 | e5220 | DOI: https://doi.org/10.25248/REAenf.e5220.2020 Página 8 de 9 


\section{REFERÊNCIAS}

1. ANDERSSON O, et al. Effect of delayed cord clamping on neurodevelopment at 4 years of age: A randomized clinical trial. JAMA Pediatrics, 2015; 169(7): 631-638.

2. ARMSON BA, et al. Umbilical Cord Blood Banking: Implications for Perinatal Care Providers. Journal of Obstetrics and Gynaecology Canada, 2005; 27(3): 263-274.

3. BAYER K. Delayed umbilical cord clamping in the 21st century: Indications for practice. In Advances in Neonatal Care, 2016; 16(1): 68-73.

4. BERTOLINI F, et al. Comparative Study of Different Procedures for the Collection and Banking of Umbilical Cord Blood. Journal of Hematotherapy and Stem Cell Research, 1995; 4(1): 29-36.

5. CHAPARRO CM, et al. Effect of timing of umbilical cord clamping on iron status in Mexican infants: a randomised controlled trial. Lancet, 2006; 367(9527): 1997-2004.

6. CHOPARD MRT, et al. Deficiência de ferro no feto e no recém-nascido. Revista Brasileira de Hematologia e Hemoterapia, 2010; 32(1): 32-37.

7. DAVANZO R, et al. Making the first days of life safer: Preventing sudden unexpected postnatal collapse while promoting breastfeeding. Journal of Human Lactation, 2015; 31(1): 47-52.

8. FAUCHER MA, et al. Midwives in India: a delayed cord clamping intervention using simulation. International Nursing Review, 2016; 63(3): 437-444.

9. FENTON C, et al. Clinical Outcomes in Preterm Infants Following Institution of a Delayed Umbilical Cord Clamping Practice Change. Advances in Neonatal Care, 2018; 18(3), 223-231.

10. MATHEW JL. Timing of umbilical cord clamping in term and preterm deliveries and infant and maternal outcomes: $A$ systematic review of randomized controlled trials. In Indian Pediatrics, 2011; 48(2): 123-129.

11. MCDONALD SJ, et al. Effect of timing of umbilical cord clamping of term infants on maternal and neonatal outcomes. Evidence-Based Child Health, 2014; 9(2): 350-444.

12. MERCER J, BEWLEY S. Could early cord clamping harm neonatal stabilisation? In The Lancet, 2009; 374(1): 377378.

13. MONDINI L, et al. Efeito Do Clampeamento Tardio Do Cordão Effects of Delayed Cord Clamping on Hemoglobin Values in Infants Born To Anemic and Non Anemic. Rev Bras Crescimento Desenvolvimento Hum, 2010; 20(2): 282-290.

14. MOUSTAFA AN, et al. Association between oxidative stress and cord serum lipids in relation to delayed cord clamping in term neonates. Lipids in Health and Disease, 2017; 16(1).

15. PHILIP AGS, SAIGAL S. When Should We Clamp the Umbilical Cord? NeoReviews, 2004; 5(4): e142-e154.

16. POPAT H, et al. Effect of Delayed Cord Clamping on Systemic Blood Flow: A Randomized Controlled Trial. Journal of Pediatrics, 2016; 178(1): 81-86.

17. TARNOW-MORDI W, et al. Delayed versus Immediate Cord Clamping in Preterm Infants. New England Journal of Medicine, 2017; 377(25): 2445-2455.

18. TIEMERSMA S, et al. Delayed cord clamping in South African neonates with expected low birthweight: A randomised controlled trial. Tropical Medicine and International Health, 2015; 20(2): 177-183.

19. WHO. recommendations Uterotonics for the prevention of postpartum haemorrhage. (2018).

20. WHO, et al. (2013). O clampeamento tardio do cordão umbilical reduz a anemia infantil. 5. 